\title{
OXÍGENO ADICIONAL EN INCUBACIÓN DEL POLLO DE ENGORDA
}

\author{
ADDITIONAL OXYGEN IN INCUBATION OF BROILER CHICKENS
}

\author{
Prado-Rebolledo, O.F. ${ }^{1}$, B.J.E. Morales ${ }^{2}$, L.J.A. Quintana ${ }^{3}$, A.M.J. González ${ }^{4}$ y M.J. Arce ${ }^{5}$
}

\begin{abstract}
${ }^{1}$ Facultad de Medicina Veterinaria y Zootecnia. Universidad de Colima. Km 40, autopista Colima-Manzanillo. Crucero de Tecomán. Colima. C.P. 28100. México. omarpr@ucol.mx

${ }^{2}$ Facultad de Medicina Veterinaria y Zootecnia. Universidad Autónoma Metropolitana. Unidad Xochimilco. México, D.F. México.

${ }^{3}$ Facultad de Medicina Veterinaria y Zootecnia. Departamento de Producción Animal: Aves. Universidad Nacional Autónoma de México. Ciudad Universitaria. México, D.F. México.

${ }^{4}$ Facultad de Medicina Veterinaria y Zootecnia. Departamento de Zootecnia Universidad de Chapingo. Estado de México. México.

${ }^{5}$ Facultad de Medicina Veterinaria y Zootecnia. Universidad Michoacana de San Nicolás de Hidalgo. Morelia Michoacán. México.
\end{abstract}

\section{PALABRAS CLAVE ADICIONALES}

Desarrollo embrionario. Incubación. Oxigenación.

\section{RESUMEN}

El objetivo de este ensayo fue evaluar el efecto de diferentes concentraciones de oxígeno $\left(\mathrm{O}_{2}\right)$ durante el proceso de incubación sobre los parámetros de incubación del pollo de engorda. Se utilizaron dos máquinas incubadoras, con capacidad cada una de 4800 huevos, que trabajan con dos distintas concentraciones de $\mathrm{O}_{2}$ (17,5 vs. $21 \%$ ) y 300 huevos pesados de forma individual los cuales se distribuyeron aleatoriamente en ambas máquinas. Se cuantificaron la pérdida de humedad, la incubabilidad, el peso y tamaño del pollo, nivel de glucemia, valor hematocrito, peso del vitelo y la mortalidad embrionaria. Con mayor concentración de $\mathrm{O}_{2}$ la incubabilidad aumentó en 2,13 puntos, la pérdida de humedad fue más eficiente, y el tamaño del pollo fue mayor en 0,44 $\mathrm{cm}$; la concentración de glucosa sanguínea se redujo $(-16,4 \mathrm{mg} / \mathrm{dl})$, se registró un mayor valor hematocrito (con $25,75 \pm 1,86 \%$ ), y una mortalidad embrionaria más alta en la etapa I de desarrollo. La adición de $\mathrm{O}_{2}$ en un $21 \%$, durante la incubación puede incrementar la incubabilidad, sin comprometer la integridad de las aves.

\section{SUMMARY}

The aim of this study was to evaluate the

Recibido: 19-2-07. Aceptado: 21-2-08.

\section{AdDitional KeYWORDS}

Embryonic development. Incubation. Oxygenation.

effect of different oxygen concentration during incubation on parameters in broiler chickens. Two hatchers with capacity of 4800 eggs were used to provide different oxygen concentration (17.5 vs. $21 \%$ ). 300 eggs were weighted and randomly distributed in both machines. The humidity loss, hatchability, weight and length of chick, blood glucose level, haematocrit, residual yolk and embryonic mortality were recorded. At the greatest oxygen concentration hatchability increased 2.13 percentage points, humidity was lost more efficiently, and the length of chicks increased 0.44 $\mathrm{cm}$; glycemia was reduce $(-16.4 \mathrm{mg} / \mathrm{dl})$, while haematocrit increased $25.75 \pm 1.86 \%$ ), being higher the embryo mortality during their first stage of development. The additional oxygen in $21 \%$ during the incubation may increase the hatchability, without compromising chick integrity.

\section{INTRODUCCIÓN}

Para mantener al embrión a una temperatura óptima de aproximadamente $37,8^{\circ} \mathrm{C}$, el exceso de calor tiene que ser eliminado. La mayoría de las incubadoras están diseñadas para mantener la temperatura del aire 
mediante ventilación forzada para permitir la pérdida de calor (French, 1997; Meijerhof and Lourens, 1999; Lourens, 2001), especialmente al final de la incubación. Las estirpes actuales de broilers requieren un ajuste en la incubación debido a que ha incrementado la producción de calor por el embrión. Este aumento no ha sido determinado con precisión, pero se sabe que producen cerca de dos veces más calor que los genotipos utilizados años atrás (Meijerhof and Lourens, 1999; Lourens, et al., 2005). La temperatura real del embrión es función de su producción de calor, el flujo de aire sobre el embrión y la humedad relativa del aire, por lo que el incremento en la producción de calor del embrión de alto rendimiento es un factor clave en la temperatura real que éste alcanza (French, 1997; Van Breach et al., 2005).

Debido a que el $60 \%$ del incremento del consumo de oxígeno del embrión se produce en el período que transcurre entre el inicio de la respiración pulmonar y el nacimiento, diferencias en la conductibilidad del cascarón y en los mismos parámetros técnicos del proceso de incubación pueden inducir cuadros de hipoxia, sobre todo en el intervalo de tiempo entre el inicio del picado interno de la cáscara y el momento del nacimiento, en el cual existe una demanda mayor $\mathrm{de}_{2}$. Este proceso de hipoxia puede desencadenar una serie de trastornos fisiológicos que pueden comprometer la vida y desempeño productivo de las aves. El objetivo del presente trabajo fue evaluar el efecto de diferentes concentraciones de $\mathrm{O}_{2}$ durante el proceso de incubación, en máquinas de una sola etapa, sobre los parámetros de incubación del pollo de engorda.

\section{MATERIAL Y MÉTODOS}

Los trabajos se efectuaron en la planta incubadora Avícolas de Colima S.A de C.V., localizada en el Estado de Colima, México, cuya ubicación geográfica es $103^{\circ} 43^{\prime} 12^{\prime \prime}$ latitud norte y $19^{\circ} 16^{\prime} 50^{\prime \prime}$ longitud oeste, a una altitud sobre el nivel del mar de 442 metros. El clima de la región está clasificado como cálido húmedo según García (1988), y descrito con una temperatura media anual de $25,3^{\circ} \mathrm{C}$ y precipitación pluvial media anual de $1007 \mathrm{~mm}$.

Se utilizaron dos incubadoras comerciales de una sola etapa de la marca HatchTech Combi $4800^{*}$, con una capacidad cada una de 4800 huevos. Estas máquinas cuentan con sensores de temperatura $\left( \pm 0,1^{\circ} \mathrm{C}\right)$, sensor de humedad relativa $( \pm 1 \%$ de HR) y sensor de $\mathrm{CO}_{2}( \pm 100 \mathrm{ppm})$; la circulación del aire es horizontal, y se controla su velocidad en las diferentes etapas del proceso de incubación. El aire pasa a través de paneles de radiación, controlados mediante la información de programación en el panel de control. Las mismas máquinas hacen la función de nacedoras.

En ambas máquinas se realizó el manejo de rutina propio de una planta incubadora, desde la recepción del huevo hasta el retiro de los pollos de las máquinas nacedoras. Se formaron dos tratamientos de 150 huevos incubables cada uno, descartando los rotos, sucios, microfracturados, deformes, etc., procedentes de reproductoras de 33 semanas de edad del mismo lote y estirpe Cobb, los cuales se pesaron e identificaron, sometidos a dos concentraciones diferentes de $\mathrm{O}_{2}(17,5$ y $21 \%$, que corresponden a 112 torr $\mathrm{pO}_{2}$ y 134 torr $\mathrm{pO}_{2}$ ). El flujo de $\mathrm{O}_{2}$ se ajustó con un fluxómetro a 13,5 1/min. La máquina a la que se le proporcionaba el $\mathrm{O}_{2}$ adicional se mantuvo con un tanque suministrador de $\mathrm{O}_{2}$ colocado adyacente a la máquina incubadora, ambas máquinas se mantuvieron a la misma temperatura durante todo el período de incubación, $37,8^{\circ} \mathrm{C}$ en el día 0, y $37,61^{\circ} \mathrm{C}$ del día $1^{\circ}$ al $8^{\circ} ; 37,50^{\circ} \mathrm{C}$ en los días $9^{\circ}-11^{\circ} \mathrm{y}$ $37,22^{\circ} \mathrm{C}$ desde el día $12^{\circ}$ al $21^{\circ}$. La humedad relativa se mantuvo en un $50 \%$ durante todo el experimento. Al $10^{\circ}$ día de incubación,

*HatchTech Incubation Technology. Gidetrom 25 P.O. Box 256 N1-3900 AG Venendaal. The Netherlands. 


\section{OXÍGENO ADICIONAL EN INCUBACIÓN DEL POLLO DE ENGORDA}

todos los huevos fueron analizados para descartar los huevos infértiles y con embrión muerto, se retiraron y contaron por charola, los cuales se abrieron para su evaluación visual y determinar la fertilidad, los huevos que fallaron se examinaron macroscópicamente de cada máquina; para determinar la infertilidad y la etapa de desarrollo embrionario en la que murieron los embriones de acuerdo al criterio de North y Bell (1993). Diariamente se monitoreó el porcentaje de $\mathrm{O}_{2}$ a través de una bomba de $\mathrm{O}_{2}$ y CO (Bacharach FYRITE Gas Analyzer $\mathrm{O}_{2} \mathrm{y} \mathrm{CO}_{2}$ Indicators) así como la temperatura ambiental interna. Las variables de respuesta fueron: La temperatura del cascarón, medida diariamente con un termómetro infrarrojo (Braun thermoscan), para asegurarse de que todos los huevos incubados tuvieran la misma temperatura; el peso individual de los huevos antes de incubar y en el momento de transferencia al día 18 , a fin de determinar la pérdida de humedad; la incubabilidad; el peso y tamaño del pollito, desde la punta del pico hasta el dedo medio sin considerar la uña, como indicadores de su calidad. A 25 de las aves de cada tratamiento, se les extrajo sangre mediante punción cardíaca y vena yugular para determinar los niveles de glucosa sanguínea y hematocrito, posteriormente se les practicó la eutanasia por desnucamiento para determinar el peso de su vitelo.

Las medias obtenidas para los distintos parámetros se compararon mediante la prue- ba de $T$ de Student $(\mathrm{p}<0,05)$ (Steel y Torrie, 1990) con el paquete estadístico SAS 7 (SAS Institute, 1996). Los datos expresados en porcentajes se transformaron a la proporción arco seno para su análisis.

\section{RESULTADOS}

Con el empleo de la mayor concentración de $\mathrm{O}_{2}$ la incubabilidad aumentó significativamente $(p<0,05)$; siendo la diferencian de 2,13 puntos porcentuales (tabla I), así como la pérdida de humedad $(\mathrm{p}<0,05)$, en 2,28 puntos. La relación peso del huevo/ peso del pollo no fue estadísticamente distinta $(\mathrm{p}>0,05)$ entre tratamientos.

El peso de los pollitos tampoco varió significativamente $(\mathrm{p}>0,05)$ entre ambos tratamientos (tabla II). Los pollos fueron de mayor tamaño cuando se les suplementó $\mathrm{O}$ observándose una diferencia mayor a 0,44 $\mathrm{cm}$. El peso del vitelo, no mostró diferencias estadísticamente significativas $(p>0,05)$. El valor del hematocrito fue significativamente distinto $(\mathrm{p}<0,05)$, siendo superior en las aves del tratamiento de adición de $\mathrm{O}_{2}(16,4$ $\mathrm{mg} / \mathrm{dl})$. La mortalidad embrionaria mostró diferencias significativas $(\mathrm{p}<0,05)$ en la etapa I, siendo mayor en el tratamiento con mayor concentración de $\mathrm{O}_{2}$, pero ya no se registraron diferencias significativas en etapas posteriores $(\mathrm{p}<0,05)$ (tabla III).

\section{DISCUSIÓN}

La incubabilidad mejoró con la adición

Tabla I. Incubabilidad, pérdida de humedad y relación huevo/pollo con y sin la adición de $\mathrm{O}_{2}$ en incubación. (Hatchability, weight loss and relation egg/chick with and without the addition of $\mathrm{O}_{2}$ during the process of incubation).

\begin{tabular}{lccc}
\hline & Incubabilidad $(\%)$ & Pérdida de humedad $(\%)$ & huevo/pollo* \\
\hline Sin oxígeno & $87,70 \pm 1,41^{\mathrm{b}}$ & $9,16 \pm 1,77^{\mathrm{a}}$ & $25,84 \pm 0,81^{\mathrm{a}}$ \\
Con oxígeno & $89,83 \pm 1,83^{\mathrm{a}}$ & $11,44 \pm 3,46^{\mathrm{b}}$ & $28,06 \pm 0,98^{\mathrm{a}}$ \\
Diferencia & 2,13 & 2,28 & 2,22 \\
\hline
\end{tabular}

abLetras distintas en la misma columna indican diferencias estadísticamente significativas $(p<0,05)$. *peso huevo/peso pollo (\%). 
PRADO-REBOLLEDO, MORALES, QUINTANA, GONZÁLEZY ARCE

Tabla II. Efecto de dos concentraciones $\mathrm{O}_{2}$ durante el proceso de incubación sobre distintas variables fisiológicas de los pollos. (Effect of two concentrations of $\mathrm{O}_{2}$ during the process of incubation on several physiological parameters of chicken).

\begin{tabular}{lccccc}
\hline & $\begin{array}{c}\text { Peso } \\
(\mathrm{g})\end{array}$ & $\begin{array}{c}\text { Tamaño } \\
(\mathrm{cm})\end{array}$ & $\begin{array}{c}\text { Vitelo } \\
(\mathrm{g})\end{array}$ & $\begin{array}{c}\text { Glucosa } \\
(\mathrm{mg} / \mathrm{dl})\end{array}$ & $\begin{array}{c}\text { Hematocrito } \\
(\%)\end{array}$ \\
\hline Sin oxígeno & $44,90 \pm 2,7^{\mathrm{a}}$ & $18,37 \pm 0,65^{\mathrm{b}}$ & $6,24 \pm 1,75^{\mathrm{a}}$ & $218,4 \pm 32,6^{\mathrm{a}}$ & $24,72 \pm 1,56^{\mathrm{b}}$ \\
Con oxígeno & $44,51 \pm 3,2^{\mathrm{a}}$ & $18,81 \pm 0,44^{\mathrm{a}}$ & $5,19 \pm 1,3^{\mathrm{a}}$ & $202,0 \pm 1,37^{\mathrm{b}}$ & $25,72 \pm 1,86^{\mathrm{a}}$ \\
Diferencias & $-0,39$ & 0,44 & $-1,05$ & $-16,4$ & 1,0 \\
\hline
\end{tabular}

ab Letras distintas en la misma columna indican diferencias estadísticamente significativas $(p<0,05)$.

de $\mathrm{O}_{2}$ lo cual indica que si los pollos pueden disponer de mayor cantidad de moléculas de $\mathrm{O}_{2}$ no se deterioran fisiológicamente al momento del nacimiento; sin embargo, hay una gran gama de factores que pueden influir en la incubabilidad. Christensen y Bagley (1988); encontraron una mejora en el número de pavipollos nacidos con la adición de $\mathrm{O}_{2}$ en máquinas incubadoras ubicadas en altitudes superiores a $2000 \mathrm{msnm}$, debido a que los pavipollos tuvieron mayor disponibilidad de $\mathrm{O}_{2}$, que les ayudó a acelerar el crecimiento de su tasa metabólica de los embriones. Cuando el embrión cambia la respiración corioalantoidea por respiración pulmonar, precisa una mayor disponibilidad de moléculas de $\mathrm{O}_{2}$ en la máquina nacedora; si el aporte de $\mathrm{O}_{2}$ no es adecuado, se puede propiciar un cuadro de hipoxia que puede desencadenar hipertensión pulmonar, que conduce a la manifestación del síndrome ascítico en la etapa de crecimiento (Julian, 2000).

La pérdida de humedad fue menor en el tratamiento sin adición de $\mathrm{O}_{2}$, a pesar de lo cual no se observaron alteraciones en los pollos que pusieran de manifiesto una insuficiente pérdida de humedad, como las indicadas por Phillips et al. (1992). Así, la tasa de pérdida de agua de los huevos (que refleja indirectamente el intercambio de gases vitales está controlada en gran medida por la humedad relativa de la máquina incubadora (Bruzual et al., 2000; Burnham et al., 2001).

La relación entre el peso del huevo/peso del pollo al nacimiento debe estar en el rango de $28-33 \%$ (Wilson, 1991). Aunque los valores obtenidos en el presente experimento se encuentran al margen y por debajo del límite inferior, los pollos se encontraron aparentemente normales. Existe una alta correlación positiva entre el peso del huevo y peso corporal de los pollos (Wilson, 1991; Suarez et al., 1997; Peebles et al., 1999). Melcalfe et al. (1981) observaron que el $\mathrm{O}_{2}$ acelera el metabolismo y desarrollo en embriones de pollo en desarrollo, por lo que el

Tabla III. Efecto de diferentes concentraciones de $\mathrm{O}_{2}$ sobre la mortalidad embrionaria e infertilidad. (Effect of different $\mathrm{O}_{2}$ concentrations on the embryo mortality and infertility).

\begin{tabular}{lccccc}
\hline & Etapa I & Etapa II & Etapa III & Etapa IV & Infértil \\
\hline Sin oxígeno & $2,35 \pm 0,8^{\mathrm{b}}$ & $0,71 \pm 0,42^{\mathrm{a}}$ & $0,73 \pm 0,33^{\mathrm{a}}$ & $0,47 \pm 0,39^{\mathrm{a}}$ & $7,94 \pm 0,53^{\mathrm{a}}$ \\
Con oxígeno & $3,70 \pm 1,0^{\mathrm{a}}$ & $0,76 \pm 0,56^{\mathrm{a}}$ & $0,81 \pm 0,28^{\mathrm{a}}$ & $0,61 \pm 0,33^{\mathrm{a}}$ & $4,19 \pm 0,29^{\mathrm{b}}$ \\
Diferencia & 1,35 & 0,05 & 0,08 & 0,14 & 3,75 \\
\hline
\end{tabular}

abLetras distintas en la misma columna indican diferencias estadísticamente significativas $(p<0,05)$.

Archivos de zootecnia vol. 58, núm. 221, p. 88. 


\section{OXÍGENO ADICIONAL EN INCUBACIÓN DEL POLLO DE ENGORDA}

embrión optimiza sus reservas de energía para sus tejidos y nace sin un deterioro metabólico que pueda poner en riesgo la integridad fisiológica de los pollos.

El tamaño de los pollos a los que se proporcionó el $\mathrm{O}_{2}$ adicional fue significativamente mayor $(\mathrm{p}<0,05)$. Ello sugiere, que al disponer los embriones de pollo de más moléculas de $\mathrm{O}_{2}$ en el momento del cambio de respiración corioalantoidea a pulmonar, el embrión utiliza sus reservas fisiológicas de energía para sus funciones metabólicas, optimizando así su desarrollo. Las temperaturas superiores a lo normal durante el proceso de incubación inducen un nacimiento prematuro de los pollitos; por el contrario, si las temperaturas se mantienen por debajo de los límites de las temperaturas de incubación se puede prolongar los nacimientos (Decuypere y Michels, 1992; French, 1997). En el presente trabajo se tomó como indicador de calidad el tamaño del pollito, y según este criterio los pollitos que tuvieron mayor disponibilidad de $\mathrm{O}_{2}$ ambiental fueron de mayor calidad (Hill, 2001).

El peso del vitelo no mostró diferencias significativas entre ambos tratamientos. Burnham et al. (2001) señalan que la humedad de la yema está fuertemente influida por la humedad relativa durante el proceso de incubación, así como por su porcentaje de lípidos, y que el porcentaje de vitelo de los pollos que provienen de reproductoras viejas es más pequeño que el de las reproductoras jóvenes. El vitelo es la principal fuente de nutrientes para el desarrollo del embrión (Yael y Sklan, 1999; Noy y Sklan, 2002), en especial al final del desarrollo embrionario, por lo que su absorción es más rápida en pollos que provienen de reproductoras jóvenes para mantener altas concentraciones de agua en los tejidos, pero declina el contenido de humedad con la edad de los embriones (Peebles et al., 1999). Por lo que los valores obtenidos en el presente estudio coinciden con los resultados obtenidos con los autores anteriores.

Los niveles de glucosa sanguínea fue- ron significativamente mayores en las aves que no tuvieron $\mathrm{O}_{2}$ adicional. Cuando las aves se encuentran en ayuno, o sufren alguna situación de estrés, utilizan las reservas de glucógeno de los tejidos (glucogenolisis) con lo que aumentan los valores de glucosa en sangre (hiperglucemia). Peebles, et al. (2005) realizaron un experimento para relacionar varios parámetros fisiológicos en pollitos recién nacidos con la restricción de nutrientes en gallinas jóvenes, observando que el peso corporal de las aves se reducía en un $19 \%$ entre las 0 y 72 h posnacimiento, sin que se produjera mortalidad. Los picos más altos de glucógeno hepático se registraron a las $24 \mathrm{~h}, \mathrm{y}$ los picos más altos de temperatura rectal y de glucosa en el plasma ocurrieron a las $48 \mathrm{~h}$, pero se observó una correlación negativa entre el peso corporal y el peso del hígado y entre el glucógeno hepático. En el presente trabajo los valores de glucosa fueron más altos en el tratamiento que no recibió la adición de $\mathrm{O}_{2}$, posiblemente debido a la utilización de las reservas de glucógeno de los tejidos para su mantenimiento.

El hematocrito mostró diferencias significativas entre tratamientos, siendo más alto en el tratamiento con $\mathrm{O}_{2}$ adicional, aunque los valores se encuentran dentro de los parámetros normales, donde no se compromete el transporte de $\mathrm{O}_{2}$ en la sangre (Julian, 2000). A pesar de que se utilizaron huevos del mismo lote de reproductoras, la infertilidad fue mayor en el tratamiento donde no se le adicionó $\mathrm{O}_{2}$, lo cual puede estar explicado por la edad, manejo y otros factores involucrados en la reproducción de las aves. La mortalidad embrionaria fue mayor en el tratamiento con más moléculas de $\mathrm{O}_{2}$ durante la etapa I de desarrollo embrionario; posteriormente ya no hubo diferencias significativas ( $p>0,05)$. La mortalidad embrionaria en los primeros días de incubación está influida por el número de días de almacenamiento y las condiciones del mismo (Brake et al., 1997). Se han registrado dos fases de incremento de la mortalidad embrionaria 


\section{PRADO-REBOLLEDO, MORALES, QUINTANA, GONZÁLEZY ARCE}

durante la incubación; la primera fase ocurre durante la primera semana de incubación y la segunda durante la última semana (Kuurman et al., 2002). En el presente trabajo únicamente se apreció un aumento de la mortalidad en la primera etapa de incubación; este incremento pudo deberse a una serie de procesos como la rápida formación vascular del embrión en desarrollo, el pico de producción de ácido láctico, los cambios originados por la eliminación de $\mathrm{CO}_{2}$ y por el inicio de la función del mesonefro de los riñones embrionarios. Al final del periodo

\section{BIBLIOGRAFÍA}

Brake, J., T.J. Walsh, C.E. Jr. Benton, J.N. Petite, R. Meijerhof and G. Peñalva. 1997. Egg handing and storage. Poult. Sci., 76: 144-151.

Bruzual, J.J., S.D. Peak, J. Brake and E.D. Peebles. 2000. Effects of relative humidity during incubation on hatchability and body weight of broiler chick from young breeder flocks. Poult. Sci., 79: 827-830.

Burnhan, M.R., E.D. Peebles, C.W. Gardner, J. Brake, J.J. Bruzual and P.D. Gerard. 2001 Effects in incubator humidity and hen age on yolk composition in broiler hatching eggs from young breeders. Poult. Sci., 80: 1444-1450.

Christensen, V.L. and L.G. Bagley. 1988.Improved hatchability of turkey eggs at high altitudes due to added oxygen and increased incubation temperature. Poult. Sci., 67: 956-960.

Decuypere, E. and H. Michels. 1992. Incubation temperature as a management tool: a review. W. Poult. Sci. J., 48: 28-38.

French, N.A. 1997. Modeling incubation temperature: The effects of incubator design, embryonic development, and egg size. Poult. Sci., 76: 124-133.

García, E. 1988. Modificaciones al sistema de clasificación climática de Köppen: para adaptarlo a las condiciones de la República Mexicana. Edit. Universidad Nacional Autónoma de México. México. D.F. 130 p.

Julian, J.R. 2000. Physiological, management and environmental triggers of the ascites syndrome: a review. Avian Pathol., 29: 519-527.

Hill, D. 2001. Chick length uniformity profiles as a de incubación, la mortalidad embrionaria puede darse por el incremento significativo de la demanda de $\mathrm{O}_{2}$.

\section{CONCLUSIONES}

La adición de $\mathrm{O}_{2}$ en un $21 \%$, durante el periodo de incubación incrementa la incubabilidad, lo que sugiere un equilibrio entre la tolerancia a la hipoxia y la posibilidad de reducir la intensidad de la respiración por unidad de masa corporal, sin comprometer la integridad de las aves.

field measurement of chick quality?. Avian Poult. Biol. Rev., 12: 188.

Kuurman, W.W., B.A. Bailey, W.J. Koops and M. Grossman. 2002. Influence of storage days on the distribution for time of embryonic mortality during incubation. Poult. Sci., 81: 1-8.

Lourens, S. 2001. The importance of air velocity in incubation. W. Poult., 17: 29-30.

Lourens, A., H. Van den Brand, R. Meijerhof and B. Kemp. 2005. Effect of eggshell temperature during incubation on embryo development, hatchability, and posthatch development. Poult. Sci., 84: 914-920.

Meijerhof, R. and S. Lourens. 1999. Embryo temperature is the key factor in incubation. $W$. Poult., 15: 42-43.

Metcalfe, J., I.E. McCutcheon, D.L. Francisco, A.B. Metzenberg and J.E. Welch. 1981. Oxygen availability and growth of the chick embryo. Respir. Physiol., 46: 81-88.

North, M.O. y D. Bell. 1993. Manual de producción avícola. $3^{a}$ Ed. Edit. Manual Moderno. México.

Noy, Y. and D. Sklan. 2002. Nutrient use in chick during the first web posthatch. Poult. Sci., 81: 391-399.

Peebles, E., L. Li, S. Miller, T. Pansky, S. Whitmarsh, A.M. Latour and D.P. Gerard. 1999. Embryo and yolk compositional relationships in broiler hatching eggs during incubation. Poult. Sci., 78: 1435-1442.

Peebles, E.D., R.W. Keirs, L.W. Bennett, T.S. Cummings, S.K. Whitmarsh and P.D. Gerard. 2005. Relationships among prehatch and

Archivos de zootecnia vol. 58, núm. 221, p. 90. 


\section{OXÍGENO ADICIONAL EN INCUBACIÓN DEL POLLO DE ENGORDA}

posthatch physiological parameters in early nutrient restricted broilers hatched from eggs laid by young breeder hens. Poult. Sci., 84: 454561.

Phillips, L., J. Brake, S. Ellner and O. Rachel. 1992. A mathematical model for estimation of broiler egg weight loss from physical dimensions and air cell size during incubation. Poult. Sci., 71: 625-630.

SAS Institute. 1996. SAS/STAT Sofware: Changes and enhancements through release 6.11. SAS Institute, Inc. Cary, NC.

Steel, R.G.D. and J.H. Torrie. 1990. Principles and procedures of Statistix. A biometrical approach. $2^{\text {nd }}$ Ed. McGraw-Hill. Singapore.
Suarez, M.E., H.R. Wilson, F.B. Mather, C.J. Wilcox and B.N. McPherson. 1997. Effect of strain and age of the broiler breeder female on incubation time and chick weight. Poult. Sci., 76: 10291036.

Van Brech, A., H. Hens, J.-L. Lemaire, J.M. Aerts, P. Degraeve and D. Berckmans. 2005. Quantification of the heat exchange of chicken eggs. Poult. Sci., 84: 353-361.

Wilson, H.R. 1991. Interrelationship of egg size, chick size, post hatching growth and hatchability. W. Poult. Sci. J., 47: 5-20.

Yael, N. and D. Sklan. 1999. Energy utilization in newly hatched chicks. Poult. Sci., 78: 17501756.

Archivos de zootecnia vol. 58, núm. 221, p. 91. 